\title{
ZUR SCHREIBUNG DER ORTSNAMEN IN DER DEUTSCHSPRACHIGEN SCHWEIZ
}

\author{
Von Johannes Hubschmid
}

Es ist allgemein bekannt, daß die mundartliche Aussprache der Ortsnamen von ihrer Schreibform oft mehr oder weniger stark abweicht. Gewisse Schreibungen spiegeln einen älteren Sprachzustand wider. Flawil, 858 Flawilare, wird heute Flowil oder Flobel gesprochen, mit langem, offenem o wie schof "Schaf» in derselben Mundart. In der Schreibung Mosnang kommt dieser mundartliche Wandel von a zu o zum Ausdruck; 854 ist Masinang belegt. Die ortsübliche Aussprache lautet hingegen Moslig (mit langem, offenem o). Ursprünglich wurden beide $\mathrm{n}$ gesprochen, dann wurde das erste infolge Dissimilation durch ein 1 ersetzt, und später schwand das $n$ der Auslautsilbe wie zum Beispiel im schweizerdeutschen zitig "Zeitung». Heute schreiben wir Binningen mit voller, Frutigen mit verkürzter Endung. Seit 1224 bis in die Mitte des 19. Jahrhunderts war noch die Form Frutingen gebräuchlich. Die Endung beider Namen wird aber gleich ausgesprochen. Auch in Olten, Meilen, Silberen, Ankenhüsli, Forenbach usw. ist das $n$ lediglich durch die Schreibtradition begründet.

Auf eine schon alte, falsche Sinndeutung weist die Form Luzern. Durch die Einführung der seit dem 11./12. Jahrhundert bezeugten klösterlichen Latinisierung Lucerna wurde aus einer ursprünglich römischen Fischersiedlung an einer Hechtreuse (romanisch luciaria, zu lucius «Hecht») eine "Leuchtenstadt»". Neben Lucerna u. ä. sind folgende Belege überliefert: Luciaria 840, Lucerra 1185, Luceria $1210^{2}$, Lucerrun 1226 , Luzeren 1285 , Luzzeren 1300 , Lucerie $1302,1331^{2}$, Lucerren 1308, 1309, Lutzerren 1329, 1331, 1336, 13513, seither (nach den Eidg. Abschieden) Lutzern u. ä. Diese auf dem gelehrten Lucerna beruhende Form verdrängte schließlich sogar die volkstümliche Aussprache des Namens.

Schüpfheim im Kanton Luzern wird gleich ausgesprochen wie Schüpfen im Kanton Bern und anderswo. Die ältesten Belege für Schüpfheim lauten Sciuphon 1247, Schüephen 1323, Schüppfen 1382; Schüpfheym taucht zum erstenmal im Jahre 1666 auf $^{4}$. LEU (1760) schreibt noch Schüpffheim oder Schüpffen ${ }^{5}$. Irgendein Kanzlist hatte in der Endung ein abgekürztes, "verdorbenes» -heim vermutet, doch zu Unrecht. In Wirklichkeit bezeichnete der Name eigentlich einen Ort «bei den Schüpfen», d. h. in der Nähe von Schöpfen oder Schuppen ${ }^{4}$. In noch jüngere Zeit fällt die Entstellung von Kirsiten in Kehrsiten. Urkundlich bezeugt sind Chirsitun 1218, Kirsitun 1283, Kirsiten 1309, Kirseton 1313, Kirsiten 16456, 1712 usw.,

1 J. U. Hubschmied: Geschichte des Kantons Luzern von der Urzeit bis zum Jahre 1500, S. 499 bis 500. Luzern 1932.

2 Luceria, -e in lateinisch geschriebenen Urkunden.

${ }^{8}$ Lutzerren u. ä. erklären sich durch reguläre althochdeutsche Entwicklung aus dem mittellateinischen Luciaria.

4 Schweiz. Idiotikon, 8, 1092; W. BRUCKNER: Schweizerische Ortsnamenkunde, S. 99. Basel 1945.

5 Allgemeines Helvetisches, Eydgenössisches oder Schweizerisches Lexicon, Bd. 16, S. 483. Zürich 1750.

- J. L. CYSAT: Beschreibung des berühmten Lucerner- oder Vierwaldstättersees. Luzern 1661. Karte. 
bis in die Mitte des 19. Jahrhunderts, und neuerdings wieder heute, auf Anregung des verstorbenen Staatsarchivars R. DURRER, im 1930 reproduzierten Übersichtsplan der schweizerischen Grundbuchvermessung. Für die erste Verunstaltung des Namens scheint LEU verantwortlich zu sein; er schreibt $(1756)$ Kirseiten ${ }^{7}$. Ihm folgt die Carte de la Suisse von W. Coxe mit Kersiten 1786; die weiteren Abwandlungen sind Kehrsiten $1811^{8}$, Kirsiten oder Kehrseiten $1840^{\circ}$, Kehrseiten, in der Volkssprache Kirscheten $1846^{10}, \mathrm{Kehrseiten} 1850,1854^{11}$. Die erste Ausgabe der Dufourkarte (1864) und die Siegfriedkarte verzeichnen Kehrsiten. Die bodenständige Aussprache, die ohne Zuhilfenahme von phonetischen Zeichen am besten durch Chirschete wiedergegeben werden kann, hört man an Ort und Stelle nur noch selten; in Luzern ist sie überhaupt unbekannt. Chirschete bedeutete ursprünglich, wie schon J. L. BrandSTETter erkannt hatte, "Kirschbaumhain»12, nicht "die. Kehrseite (des Berges)».

Diese Beispiele, die sich noch leicht vermehren ließen, sind nicht etwa "volksetymologische» (im eigentlichen Sinn des Wortes), sondern gelehrte, irrtümliche Sinndeutungen und Umgestaltungen; sie sind meist in den Schreibstuben der Kanzleien entstanden. Insbesondere in der modernen Zeit, wo das geschriebene Wort einen so starken Einfluß ausübt, dringen solche entstellte Formen leicht in die allgemeine Umgangssprache.

Schließlich finden wir Ortsnamen, die in Anlehnung an die neuhochdeutsche Schriftsprache ohne Verfälschung des ursprünglichen Sinnes teilweise oder ganz verhochdeutscht wurden. Wir erinnern an Schreibungen, wie Reuti, Reute oder Rüte neben den häufigeren Rüt(t)i, Ritti, an Scheuer, Scheur (auch als zweites Namenglied), neben mehr als doppelt so vielen Schür, an Mauer (20), Mauern (3), Maur (11), Mauren (7), neben Mur (22), Muren (4), Mura (2 Beispiele ${ }^{13}$ ), an die vorherrschende Schreibung Grubi (statt Gruebi) im Berner Oberland oder an Kühliberg (statt Chielibärg) im Kanton Uri. Grubi, Grübli, Kühli sind vergleichbar mit in hochdeutschem Text gebrauchten Gattungswörtern, wie Rübli, Küchli, Tüchli.

Kürzlich sind diese Mißstände in der Ortsnamenschreibung von ED. IMHoF kritisiert worden ${ }^{14}$. Der alte Grundsatz, die Schreibung nur dann abzuändern, wenn die irrtumsfreie Verständigung zwischen dem Einheimischen und dem ortsfremden Kartenbenützer in Frage gestellt sei, ist verfehlt; das geht auch aus der Lektüre des Aufsatzes von IмноF hervor. Wer möchte bezweifeln, daß die beanstandeten, von den Kanzleien übernommenen Kartenschreibungen Häusli, Grübli, Rüte, Farnern, um nur einige der von IMHOF erwähnten verbesserungsbedürftigen Schreibformen zu nennen, die irrtumsfreie Verständigung hindern würden?

IMHOF kritisiert auch die noch 1947 offiziell gültige Instruktion der Eidg. Landestopographie: "Ortsnamen, welche ohne weiteres in die Schriftsprache übertragen

7 Lexicon, Bd. 11, S. 113.

8 J. Businger: Die Stadt Luzern und ihre Umgebungen, S. 245. Luzern 1811.

- J. G. Erex: Anleitung, auf die nützlichste und genußvollste Art die Schweiz zu bereisen, 7. Auflage, S. 316. Zürich 1840.

$10 \mathrm{~J}$. J. Leurhy: Geographisch-statistisches Handlexicon des Schweizerlandes. Zürich 1846.

11 Karte vom Kanton Luzern; Wandkarte vom Kanton Luzern.

12 Wöchentliche Unterhaltungen, Beilage zum Luzerner Tagblatt 1869, S. 44; vgl. ferner W. BRUCKNER: Vox Romanica 1, 1936, S. 242, Basler Nachrichten 1946, Nr. 216.

13 Nach dem auf der Eidg. Landestopographie befindlichen Ortsnamenkatalog des Siegfriedatlasses.

14 "Die Ortsnamen in den amtlichen Plänen und Karten", Sonderdruck aus der Schweizerischen Zeitschrift für Vermessungswesen und Kulturtechnik 1945, Heft 5-9. 
werden können und an Ort und Stelle in dieser Schreibweise gebraucht werden, bekannt und verständlich sind, sind in der Schriftsprache wiederzugeben. Namen, die jedoch nur im landläufigen Dialekt existieren und nur in dieser Form bekannt und verständlich sind, müssen in Dialektform geschrieben werden.» Er führt dazu aus: "Verhängnisvoll ist das Festhalten an der grundsätzlichen Bestimmung, wonach die Schreibform der Karte stets abhängig gemacht werden soll von einer schon vorhandenen orts üblichen Schreibweise, also auch dann,wenn diese sprachlich unbefriedigend oder falsch ist. Diese Bestimmung trug die Hauptschuld am "Schprachschund' unserer Nomenklatur. Sie erniedrigte die Karte zum Sammelkratten all der zweifelhaften Elaborate sprachlich unkundiger Schreiber und Kanzlisten des letzten Jahrhunderts. Selbst Modetorheiten, Prinzipienlosigkeit und Entwurzelung wurden damit sanktioniert und verbrieft ${ }^{\mathbf{1 5}}$.»

Mit Recht stellt Iмноғ die Frage, was eigentlich bei den Ortsnamen unter "Schriftsprache» zu verstehen sei, ohne jedoch selbst eine eindeutige Antwort geben zu können. Fast auf jeder Seite seiner Abhandlung treffen wir die Ausdrücke "Mundart» "Schriftsprache», die vorerst einer klaren Definition bedürfen.

Unter "Schriftsprache» verstehen wir ganz allgemein die für den schriftlichen Verkehr bestimmte und als solche in den einzelnen Epochen anerkannte Sprachform, die geschriebene Sprache ${ }^{\mathbf{1 6}}$. "Die Schriftsprache ist auf dem Mutterboden der Mundart erwachsen. Zu keiner Zeit hat das landschaftliche Element in die Schriftsprache zu fließen aufgehört ...; daher auch die vielgenannten schweizerischen Ausdrücke, die SCHILler in seinen ,Tell eingeführt hat ${ }^{17}$. Wenn Ortsnamen, wie Zürich, Albis, Etzel, Köniz, Zollbrück, Brugg, Egg, Bödeli, Wolhusen, Farnern neben Farneren, im Kanton Fribourg Farnera usw., im schriftlichen Verkehr gebräuchlich sind, so sind dies schriftsprachliche Formen, gleichgültig, ob sie Duden verzeichnet ( $Z \ddot{u ̈ r i c h}$ ) oder nicht, ob ihre mundartliche Aussprache von der angenommenen Schreibung erheblich abweicht (Züri, Chüniz, Wolhuse usw.) oder weitgehend entspricht (Albis, Egg, Bödeli usw.). Auch verbesserte, auf der ortsüblichen Aussprache beruhende Schreibungen, wie Egg (statt Eck, wie zuweilen in Anlehnung an das Wort Ecke geschrieben wird ${ }^{18}$ ), Zollbrügg, Farnere werden "schriftsprachlich», sobald sie eingeführt und allgemein gebräuchlich geworden sind.

Unter "Mundart» verstehen wir, im Gegensatz zur Schriftsprache, die gesprochene Sprache. Die geschriebene Sprache der Dialekt- oder Mundartdichtung ist weder Mundart im eigentlichen Sinn noch Schriftsprache; Schriftsprache ist sie deshalb nicht, weil diese Schreibform, die ganz andern Zwecken dient, weder allgemein gebräuchlich ist noch je allgemein gebräuchlich wird. Die im wesentlichen aus Gattungswörtern bestehende Schriftsprache der Bücher und Zeitungen usw. wird immer, sei es in der heutigen oder in einer zukünftigen Form, auf dem gesamten von ihr beherrschten Gebiet, im Lautstand mehr oder weniger einheitlich sein. Für die eńg mit dem Boden verwachsenen Ortsnamen ist eine solche Vereinheitlichung nicht notwendig; darum entwickelte sich ihre Schreibform seit jeher zum Teil in anderer Richtung als die Schreibung der entsprechenden deutschen Gattungswörter. Wenn auch heute die Schreibung $\mathrm{Halde}(\mathrm{n})$ im Siegfriedatlas bedeutend häufiger bezeugt ist als Halta (4), Halte (4), Halten (142 Belege), so zeigen doch die 150 Beispiele mit t, $\mathrm{daß}$ sich die Verhochdeutschung zu Halde nicht überall durchsetzen konnte ${ }^{19}$.

15 Iмноғ: а. а. O., S. 12.

18 W. Henzen: Schriftsprache und Mundarten, S. 35. Zürich 1938.

17 W. HENZEN: a. a. O., S. 18.

18 Die Schreibung Eck (auch als zweites Namenglied) ist im Siegfriedatlas $37 \mathrm{mal}$ belegt.

10 Halde entspricht häufig zwar auch der Mundart; inwieweit Schreibungen von Halde statt älterem und mundartnäherem Halte stehen, haben wir nicht untersucht. 
Auch die schon erwähnten Formen Egg, Eck und noch manche andere Namen sind Zeugen für die von den entsprechenden Gattungswörtern verschiedene Behandlung der Ortsnamen im schriftlichen Gebrauch. Eine konsequente Verhochdeutschung der Ortsnamen in Anlehnung an den geschriebenen Wortschatz der Bücher ist daher nicht durchführbar. Die bisher mit der Örtlichkeit verbundenen Namen würden zu abstrakten Begriffen.

Eine Abgrenzung von "schriftsprachlich» und «mundartlich» geschriebenen Namen ist in dieser Formulierung sinnwidrig. Vielmehr werden die einen Ortsnamen in Anlehnung an die mittelhochdeutsche (Kruteren), die andern in Anlehnung an die neuhochdeutsche Schreibtradition geschrieben (Krautern); in Anlehnung an die heutige Aussprache müßte die Form Chrutere lauten. Eine Dreiteilung des Namengutes in Namen, die nach mittelhochdeutscher und neuhochdeutscher Tradition und in Anlehnung an die Mundart geschrieben werden sollten, ist aber zu kompliziert und daher nicht durchführbar. Schon eine auch nur einigermaßen geregelte Zweiteilung ist unmöglich, selbst wenn man auf dem Mittelhochdeutschen beruhende Formen gelten lassen würde. Aber gerade ImHoF hebt zutreffend hervor, daß heute erstellte amtliche Pläne und Karten soweit als möglich den heutigen Zustand zu geben haben. "Auch die Sprache ist nichts dauernd Erstarrtes. Sie hat sich stets umgeformt und wird weiterhin neue Formen annehmen. Es ist daher grundsätzlich falsch, in der Gegenwartskarte erloschene Namenformen wieder herstellen zu wollen . . . ${ }^{20}$ » Es ist also unzweckmäßig, verhochdeutschte Namen, wie Krautern, durch ein nach mittelhochdeutscher Art gebildetes Kruteren zu ersetzen, oder Grübli in den sogenannten entrundenden Mundarten (gesprochen Griebli in Uri, Unterwalden, Grindelwald, im Hasli, Wallis usw.) durch ein der Mundart fremdes Grüebli.

Die bisherige, sprachlich unbefriedigende, regellose Ortsnamenschreibung läßt sich nach einem bestimmten Prinzip nur verbessern, wenn die Ortsnamen grundsätzlich soweit dies praktisch möglich ist - in Anlehnung an die lebende Sprechform geschrieben werden. Der ursprüngliche Sinn der Namen wird dadurch für denjenigen, der die Eigentümlichkeiten der Mundart nicht kennt, zwar zuweilen verdunkelt; doch ist damit praktisch kein Nachteil verbunden. Wer die Ortsnamen eines Gebietes studieren will, um daraus Schlüsse für die Erkenntnis der (früheren) Bodengestalt, der Siedlungs- und Kulturgeschichte zu ziehen, muß sich ohnehin an Ort und Stelle begeben; er wird dabei bald merken, daß man zum Beispiel im Kanton Schaffhausen Raa, im Kanton Appenzell Rään (bisher Rähn geschrieben) statt «Rain» sagt. Die irrtumsfreie Verständigung mit dem Einheimischen wird bei dieser Schreibung keineswegs gefährdet - sie wird im Gegenteil erleichtert. Auch ist es für jedermann leichter, aus der Sprechform die richtige Schreibform abzuleiten, sobald man weiß, daß die Ortsnamen in Anlehnung an die Mundart geschrieben werden. Die von uns vorgeschlagene Orthographie ist möglichst einfach und folgt zum Teil bewährter Tradition. Eine gewisse Unsicherheit kann höchstens in der Ühergangszeit auftreten. Alles ist nur eine Sache der Gewohnheit.

Eine die örtliche Mundart nicht berücksichtigende, möglichst einheitliche, moderne «schweizerdeutsche» Schreibung der Ortsnamen stand noch nie zur Diskussion; nicht einmal E. BAER, der Verfasser des allgemein abgelehnten Buches «Alemannisch. Die Rettung der eidgenössischen Seele» (Zürich 1936) ist dafür eingetreten ${ }^{21}$. Die Schaffung einer solchen künstlichen, einheitlichen schweizerdeutschen Ortsnamenschreibung würde in gleicher Weise allgemein abgelehnt; es wäre der erste Schritt zu einer selbständigen, vom Neuhochdeutschen verschiedenen allgemeinen schweizerischen

20 IMHOF: a. a. O., S. 13.

21 In einer Zuschrift an die Eidg. Landestopographie vom 5. Januar 1937. 
Schriftsprache. Wir sind überzeugt, daß unser davon abweichender Vorschlag, die Schreibung der Ortsnamen in Anlehnung an die ortsübliche Mundart, nicht nur praktisch durchführbar ist, sondern. von allen bisherigen, auf diesem Gebiet unternommenen Bestrebungen am meisten Vorteile und am wenigsten Nachteile hat.

Geographen, Volkskundler und Ortsnamenforscher haben schon vor einiger Zeit in ihren Arbeiten Örtlichkeiten mit den einheimischen, volkstümlichen Namen benannt. Selbst in fortlaufendem deutschem Text stören solche Formen nicht im geringsten. Beim Ausziehen verschiedener Werke haben wir folgende Stellen notiert: «in der Nähe der Gegend uf der Hirti » 22; "die waldige, geröllreiche Halde heißt Bä rost a mpf »..., "eine glatte, steile, mit hochwachsenden Pflanzen bewachsene Halde, die ChrütSchlüho»..., «sehr gefährlich ist nun der folgende WyB Grabo» ${ }^{23}$; die meisten großen Lawinenschläge heben sich in der Waldkarte deutlich als Lärchenbestände ab, so zum Beispiel im Uiflengenwald »..., "den hintersten Teil des Gorpäwaldes »..., "der Arvenwald in der Arbschluichen (Arbschlucht)»..., «auf dem Arbä »24; «an der Schüflu, einem kleinen, abgelegenen Weiler» ${ }^{25}$; «sie kamen uff d'Bäch und erblickten die ersten Gemsen ${ }^{26}$; "auf der uisdru Furu bei der Hockenalp standen früher Alphütten»27; "Ortsnamen, wie hinder der School, hinder em Steckli, in der Diefi und uf der Lys, die zum großen Teil infolge der baulichen Veränderungen der Stadt verloren zu gehen drohen ${ }^{28}$.

Jede Abweichung von diesen auf der Aussprache beruhenden Schreibungen, jede «Normalisierung», sei es in der Richtung nach einer altertümlichen schweizerischen Gemeinsprache oder in Anlehnung an das Hochdeutsche, ist zu verwerfen. Nicht nur würden solche künstlichen Bildungen von der einheimischen Bevölkerung ohne weiteres abgelehnt, insbesondere in Gebieten, wo viele Ortsnamen kaum je geschrieben wurden - es stellte sich auch die Frage nach dem Grad der Normalisierung. Sollte man zum Beispiel statt Uiflengenwald Üf-, Uf-oder Auflengenwald, -längenwald einführen? Statt Schüflu an der Schüfla, Schüflen, Schufla, Schuflen, Schauflen oder an der Schaufel, statt auf der uisdru Furu ein "gemeinverständlicheres» üßren Furen oder äußeren Furen, auf der äußeren Furche schreiben? Wenn irgendwo, so hat hier der Satz Imнofs Geltung, daß durch Konstruktionen, die nicht den heutigen Sprachzustand wiedergeben, "Unsicherheit und-Uneinigkeit nicht behoben, sondern ins Uferlose gesteigert würden» ${ }^{29}$. Durch Verschriftsprachlichungen, wie Weißer Graben, Äußere Furche usw., wird der Ortsname zum abstrakten Begriff. In einem Satz "ein gleiches Haus stand uf de Bächa»" ${ }^{\mathbf{3 0}}$ ist es ohne weiteres klar, daß es sich um eine bestimmte Örtlichkeit handelt. Bei einer Schreibung "auf den Bächen» kommt dies nicht recht zum Ausdruck. In gleicher Weise bestätigte man uns im Val d'Illiez, daß eine auf dem Übersichtsplan mit Pierre

22 R. Kirchgraber: Das Gebiet des ehemaligen Hochgerichtes Vier Dörfer, S. 98. Diss. Zürich 1923.

${ }^{23}$ F. G. Strabler: Ob den Heidenreben, S. 42. Zürich 1901.

24 H. Lexbundgur: Wald- und Wirtschaftsstudien im Lötschental, S. 28, 29, 107. Diss. Zürich, Bern 1938.

${ }_{25}$ Walliser Sagen, herausgegeben vom Historischen Verein von Oberwallis, Bd. 1, S. 154. Brig 1907.

${ }^{26}$ Sagen aus Uri. Aus dem Volksmund gesammelt von Joseph Müller, herausgegeben durch HanNs BäChtold-StäUbl, Bd. 2, S. 160. Basel 1929.

27 F. G. STEBLER: Am Lötschberg, S. 99. Zürich 1907.

28 W. BRUCKNER: Sonntagsbeilage zur Allgemeinen Schweizer Zeitung, S. 151. Basel 1898.

29 IмноF: a. a. O., S. 13.

so J. R. Stoppel: Das Hochtal Avers, S. 66. Zofingen 1938. 
grosse benannte Ortlichkeit eine ungeschickte Französisierung sei. Auch wenn man französisch spreche, brauche man die alte, auf der Mundart beruhende Form Pièra groussa; Pierre grosse sei zu unbestimmt, zu abstrakt, denn "des pierres grosses il y en a partout». Wie sehr zuweilen der Sinn und der tatsächliche Gebrauch eines Ortsnamens mißachtet wird, zeigen auch folgende Belege: «zwischen Schaplerstafel und Beschissene Matte» ${ }^{31}$, "so östlich von Neue Hütte»" ${ }^{32}$, "an den Ufern des Rhein " ${ }^{33}$.

IмноF betont verschiedentlich, es sei im Prinzip weder Aufgabe der Karte noch der Sprachforschung, Gebräuche abzuändern ${ }^{34}$. Doch besteht heute, wie wir aus zahlreichen Zuschriften entnehmen müssen, im Volk ein allgemeines Bedürfnis, die Namenschreibung grundsätzlich zu revidieren. Ist es da nicht Aufgabe der Eidg. Vermessungsdirektion und der Eidg. Landestopographie, diesen Wünschen entgegenzukommen? Die Schreibung der Ortsnamen ist eine kulturelle Angelegenheit. Nicht bloß der Techniker, der Plan- und Kartenersteller hat sich damit zu befassen. Die Ortsnamen werden nicht bloß von Plänen und Karten verzeichnet. Ebenso maßgebend sind die Wünsche der Kartenbenützer und der mit dem Heimatboden und Volkstum enger verwachsenen Kreise (Lehrer, Volkskundler usw.). Unsere Behörden haben die öffentlichen und allgemeinen Interessen zu wahren und zu vertreten. Nur auf diesem Wege ist eine gute, Volk und Staat dienende Lösung in der Ortsnamenschreibung erreichbar ${ }^{\mathbf{3 5}}$.

31 A. BrNz: Verbreitung der wildwachsenden Holzarten im Binnental (Kt. Wallis), S. 18. Bern 1908.

32 A. BrNz: a. a. O., S. 28.

${ }^{33}$ Als sprachwidrige Form "aus gelehrten Büchern" zitiert von W. BRUCKNER: Úber Entstehung und Geschichte unserer Ortsnamen, S. 3. Basel 1937.

34 Iмноғ: a. a. O., S. 7, 9, 30.

${ }^{35}$ Als Ergänzung zu den hier gebotenen Ausführungen vergleiche man die bei IмноF, a. a. O., S. 31, zitierte Literatur sowie die Aufsätze von J. HuBschmid in der Schweizerischen Zeitschrift für Vermessungswesen und Kulturtechnik 1947, S. 1-7, der Schweizerischen Lehrerzeitung 1947, S. 112, den Zeitschriften "Die Schweiz", April 1947, S. 25-26, und "Onomastica" (Actes du 2e Congrès international de toponymie et d'anthroponymie, Paris 1947).

\section{L'ORTHOGRAPHE ACTUELLE DES NOMS DE LIEUX DE LA SUISSE ALLEMANDE}

L'orthographe actuelle des noms de lieux de la Suisse allemande reflète, en partie, des formes de chancellerie traditionnelles qui s'expliquent par le moyen-haut-allemand; quelquefois on a changé, par une interprétation erronée, leur forme originaire. Pour d'autres noms, la forme écrite a été adaptée au système phonique de la langue littéraire allemande d'aujourd'hui, mais cette adaptation ne fut pas complète, même là où l'on aurait pu le faire. Si l'on veut trouver un système d'orthographe pratique pour les noms de lieux, il faudra choisir une forme qui ne s'écarte pas trop de la forme dialectale. Cette solution trouve, en général, l'assentiment de toutes les classes de la population.

\section{L'ORTOGRAFIA ATTUALE DEI NOMI DI LUOGO DELLA SVIZZERA TEDESCA}

L'ortografia attuale dei nomi di luogo della Svizzera tedesca riflette, parzialmente, forme di cancelleria tradizionali che si spiegano dal medio alto tedesco. Qualche volta la forma originaria di un nome fu cambiata per una interpretazione erronea. Per altri nomi di luogo, la forma scritta fu adattata al sistema fonetico della lingua letteraria tedesca moderna; ma questo adattamento non fu completo, anche là dove sarebbe stato possibile farlo. L'unico sistema di grafia pratico per i nomi di luogo sarà quello: bisogna scegliere una forma che non si scosta troppo dalla forma dialettale. A questa soluzione consentono, generalmente, tutte le classi della popolazione. 